\title{
SALUD PÚBLICA E INVESTIGACIÓN: LA AGENDA PENDIENTE
} PUBLIC HEALTH AND RESEARCH:THE PENDING AGENDA

\author{
Alonso Soto ${ }^{1, a}$, Zuño Burstein ${ }^{1, b}$
}

El presidente electo Pedro Pablo Kuczynski ha mostrado un interés particular en la mejora del sector Salud desarrollando acciones orientadas al ideal de un país en el que la salud sea un derecho real. Para ello, consideramos necesario afianzar los logros obtenidos, enmendar y aprender de los errores y generar evidencia de calidad que permita tomar eficientemente nuevas decisiones. Frente a ello, nos permitimos señalar algunos aspectos que pueden contribuir a construir una hoja de ruta con intervenciones prontas y efectivas. Dada la naturaleza de nuestra revista puntualizaremos temas de Salud Pública y relacionados al fomento de la investigación en ciencias biomédicas, proponiendo en lo posible alternativas de solución.

\section{RETOS EN SALUD PÚBLICA}

El país enfrenta una creciente demanda de atención, no solo en enfermedades infecciosas, sino de enfermedades crónicas no transmisibles. Entre estas últimas, la patología cardiometabólica y los problemas de salud mental son particularmente relevantes. El incremento en la prevalencia de diabetes, hipertensión y obesidad representa una gran preocupación. Se calcula que el $7 \%$ de la población peruana es diabética (habiéndose duplicado su prevalencia en Lima en la última década) y que el $40 \%$ de diabéticos desconocen su diagnóstico ${ }^{(1)}$. La hipertensión ha incrementado su prevalencia del 23 al $27 \%{ }^{(2)}$ y la prevalencia de obesidad se estima en $17 \%{ }^{(3)}$. Estas alarmantes cifras, asociadas al incremento de la esperanza de vida (10\% de la población peruana son adultos mayores) generarán en los próximos años una previsible "epidemia" de complicaciones tales como infartos, desórdenes cerebrovasculares e insuficiencia renal, condiciones catastróficas para el paciente, la familia y el estado. Enfrentar esta "epidemia" necesita un abordaje multifactorial que incluya la promoción de una cultura de alimentación saludable desde los colegios, fomentando el consumo de frutas y verduras, y restringiendo la venta de dulces y bebidas con alto contenido de azúcar. Esto debe ser reforzado con la urgente implementación de la reglamentación de la ley Ley de Alimentación Saludable, inexplicablemente postergada, y se debe considerar crear un impuesto a comidas no esenciales de alta densidad calórica, medida que ha logrado reducir hasta en $10 \%$ su consumo en México ${ }^{(4)}$. Asimismo, es necesaria la intervención temprana a través de consultorios que abarquen la salud cardiometabólica en forma global desde la atención primaria.

Con respecto a la salud mental, contribuyen notablemente los problemas generados por el consumo de alcohol, adicción al tabaco y los juegos de azar, pero lobbies empresariales han logrado, hasta el momento, impedir tomar acciones concretas. El tabaquismo es ciertamente un problema de salud, no solo en términos de adicción, sino además de su probado efecto en la génesis de neoplasias y eventos cardiovasculares. La generación de impuestos adicionales al consumo de bebidas alcohólicas, tabaco y juegos de azar no son solo un elemento disuasivo para estas adicciones, sino posibles fuentes de ingreso para incrementar el presupuesto destinado a atender problemas de salud mental. De igual o mayor importancia, la eliminación de cualquier tipo de publicidad de estos productos y de los juegos de azar debe ser una de las primeras acciones a tomar.

Entre las enfermedades infectocontagiosas, la tuberculosis continúa siendo un problema de salud pública (que involucra entre sus afectados también al personal de salud) cuyos indicadores epidemiológicos se han mantenido estacionarios durante la última década pese a la indudable mejora de la macroeconomía y el notable incremento del presupuesto destinado al programa presupuestal respectivo. Sin duda, el control de la tuberculosis rebasa las acciones del sector Salud e incluye el acceso a saneamiento

\footnotetext{
Revista Peruana de Medicina Experimental y Salud Pública. Instituto Nacional de Salud. Lima, Perú.

Editor científico, ${ }^{\mathrm{b}}$ Director

Correo electrónico: sotosolari@gmail.com

Recibido: 12/07/2016 Aprobado: 20/07/2016
}

Citar como: Soto A, Burstein Z. Salud pública e investigación: la agenda pendiente. Rev Peru Med Exp Salud Publica. 2016;33(3):399-400. doi:10.17843/ rpmesp.2016.333.2317 
básico, reducción del hacinamiento y eliminación de inequidades sanitarias y sociales. Hasta no lograr estas metas, la eliminación de la tuberculosis, entusiastamente promovida por la OMS ${ }^{(5)}$ parece ser una utopía. El transparentar la información epidemiológica, incluyendo la realización de estudios de prevalencia o de riesgo anual de infección tuberculosa, así como la generación y evaluación de estudios de impacto de intervenciones articuladas basadas en evidencia (intervenciones educativas, implementación de métodos rápidos de diagnóstico), deben ser prioritarios. Otros problemas relacionados con enfermedades infecciosas involucran la creciente resistencia antibiótica influenciada tanto por prescripción irracional como por la dispensación indebida por dependientes farmacéuticos; la amenaza de reemergencia de enfermedades como la lepra, peste, fiebre de Oropuche, rabia silvestre o fiebre amarilla, y el creciente impacto de nuevos virus como el zika y chikungunya, además de la propagación del dengue, entre otras.

\section{INVESTIGAR PARA PROTEGER LA SALUD}

Las políticas de salud en escenarios de recursos limitados deben basarse en evidencia de calidad que permita la asignación de los escasos recursos disponibles a aquellas intervenciones costo-efectivas. Estas evidencias deben basarse en investigación de calidad para lograr una solución eficiente de nuestros problemas. Por ello, la construcción de un sistema nacional de investigación en Salud con participación de los estamentos gubernamentales y la academia, representada por las diversas sociedades científicas y las universidades, es otro de los retos que debe ser asumido con una visión de mediano y largo plazo. Se deben definir prioridades de investigación que sean traducidas en estudios de intervención adecuadamente diseñados y con evaluaciones de impacto apropiadas. Se debe contar con financiamiento gubernamental estableciendo alianzas estratégicas con centros de investigación, fundaciones y universidades nacionales y extranjeras promoviendo el desarrollo de líneas de investigación y la formación de recursos humanos competentes. Pese a esta necesidad de formación, se ha eliminado la realización de trabajos de investigación como requisito para la obtención del título de especialista ${ }^{(6)}$. Dicho proceso se encontraba embalsado por los ineficientes procesos universitarios y la escasa calidad de los cursos de investigación durante el residentado. Sin embargo, eliminar la investigación como elemento esencial en la formación del médico especialista, y de los profesionales de la salud en general, es una decisión poco atinada, que debe ser prontamente corregida

Luego de esta mención a aspectos puntuales en salud pública e investigación, hacemos un llamado a la reflexión sobre el futuro del sistema de salud peruano. La innecesaria fragmentación del mismo, con un acceso inequitativo a la salud y brechas salariales absurdas dependiendo del tipo de sistema u organización de salud a la que pertenezcan pacientes y personal sanitario, es un problema histórico y cuya solución suele ser considerada utópica. La generación de un sistema que unifique a las instituciones del Ministerio de Salud y la seguridad social, debe ser debatida y adecuadamente planificada a fin de lograr un acceso a la salud solidario, justo y equitativo. Citando a Ottersen et al: La salud es una condición previa, resultado e indicador de una sociedad sostenible, y debe ser adoptada como un valor universal y un objetivo social y político compartido por todos ${ }^{(7)}$. Para ello, es necesario corregir la asimetría de las reformas sanitarias que han privilegiado la salud recuperativa en menoscabo de la salud pública (entendida como salud colectiva), así como brindar atención especial a la formación, capacitación continua, especialización y a la estabilización laboral con remuneraciones salariales adecuadas de los profesionales del sector Salud.

Finalizamos expresando nuestro reconocimiento a las autoridades gubernamentales e institucionales por el apoyo y estimulo otorgado a esta publicación de origen estatal que se ha ubicado como la mejor Revista médicocientífica peruana y una de las mejores latinoamericanas, siendo un ejemplo a seguir para otras publicaciones científicas nacionales. Exhortamos a las autoridades venideras que continúen con el apoyo proporcionado en beneficio de la salud y ciencia de nuestro país.

\section{REFERENCIAS BIBLIOGRÁFICAS}

1. Seclen SN, Rosas ME, Arias AJ, Huayta E, Medina CA. Prevalence of diabetes and impaired fasting glucose in Peru: report from PERUDIAB, a national urban population-based longitudinal study. BMJ Open Diab Res Care 2015;3(1):e000110. doi:10.1136/ bmjdrc-2015-000110.

2. Segura L, Agusti R, Ruiz E, Investigadores del Estudio. Factores de Riesgo de las Enfermedades Cardiovasculares en el Perú II. Estudio TORNASOL II comparado con TORNASOL I después de cinco años. Rev Peru Cardiol. 2013;34(1):5-59.
3. Instituto Nacional de Estadística e informática. Perú: Enfermedades transmisibles y no transmisibles 2014. Lima: INEI; 2015.

4. Batis C, Rivera JA, Popkin BM, Taillie LS. First-Year Evaluation of Mexico's Tax on Nonessential Energy-Dense Foods: An Observational Study. PLoS Med. 2016;13(7):e1002057. doi:10.1371/ journal.pmed.1002057.

5. World Health Organization. The End TB strategy [Internet]. Geneva: WHO; 2015 [citado el 21 de Julio de 2016]. Disponible en http://www.who.
int/entity/tb/post2015_TBstrategy. pdf ?ua $=1$

6. Valle R, Perales A. Nueva normativa de titulación en el residentado médico en el Perú: problemas y perspectivas. Rev Peru Med Exp Salud Publica. 2016;33(2):357-61. doi: 10.17843/ rpmesp.2016.332.2142

7. Ottersen OP, Dasgupta j, Blouin C, Buss P, Chongsuvivatwong V, Frenck $\mathrm{J}$, et al. The political origins of health inequity: prospects for change. Lancet. 2014;383(9917):630-67. doi: 10.1016/ S0140-6736(13)62407-1. 